\title{
Three-dimensional pathological size assessment in primary breast carcinoma
}

\author{
Uwe Güth · Denise Brenckle · Dorothy Jane Huang • \\ Andreas Schötzau · Carsten Thomas Viehl · Holger Dieterich • \\ Wolfgang Holzgreve $\cdot$ Edward Wight $\cdot$ Gad Singer
}

Received: 5 March 2008/Accepted: 25 June 2008/Published online: 12 July 2008

(C) Springer Science+Business Media, LLC. 2008

\begin{abstract}
Maximal tumor diameter (MD) is traditionally an important prognostic factor in breast cancer. It must be questioned, however, how well a one-dimensional parameter alone can represent the actual morphologic condition of a three-dimensional body. Along with the pathologically assessed MD and two perpendicular diameters (PDs) of a lesion, eccentricity (EF) and the three-dimensional parameters tumor volume (TV) and surface area (TSA) of 395 ductal invasive breast carcinomas of limited size $(10-40 \mathrm{~mm})$ were calculated. The dependent prognostic variable was axillary lymph node involvement (ALNI). MD, TV and TSA area were highly significant predictors of ALNI; these variables had similar levels of prediction
\end{abstract}

U. Güth $(\varangle) \cdot$ D. Brenckle · D. J. Huang · W. Holzgreve .

E. Wight

Department of Gynecology and Obstetrics, University Hospital

Basel (UHB), Spitalstrasse 21, 4031 Basel, Switzerland

e-mail: ugueth@uhbs.ch

A. Schötzau

JPS Institute for Biomathematics, Malzgasse 9, 4052 Basel,

Switzerland

C. T. Viehl

Department of Surgery, University Hospital Basel (UHB),

Spitalstrasse 21, 4031 Basel, Switzerland

H. Dieterich

Women's Hospital and Breast Center Rheinfelden,

Therese-Herzog-Weg 2, 79618 Rheinfelden, Germany

G. Singer

Institute of Pathology, University Hospital Basel (UHB),

Schönbeinstrasse 40, 4031 Basel, Switzerland

G. Singer

Institute of Pathology, Kantonsspital Baden AG, 5404 Baden,

Switzerland accuracy (univariate analyses: MD: $P=0.0003$, TV: $P=0.0009$, TSA: $P<0.0001$; multivariate analyses: MD: $P=0.0018$, TV: $P=0.0109$, TSA: $P=0.0009$; pseudo R-squared values: MD: 0.42, TV: 0.39, TSA: 0.39). Despite certain variations in tumor shape, TV and TSA with similar MD, there is no evidence that threedimensional pathologic measurements (TV/TSA) are more precise prognostic predictors of ALNI compared to the one-dimensional measurement alone.

Keywords Breast carcinoma - Prognosis - Tumor size . Tumor volume Tumor surface area .

Three-dimensionality

\section{Introduction}

Tumor size is traditionally one of the most sensitive and powerful indicators of prognosis in breast cancer, second only to the presence of nodal metastasis in importance [1]. Thus, it is a critical morphologic feature in staging. However, the question remains as to how well a onedimensional assessment of the largest tumor diameter alone can represent the actual morphologic condition of a threedimensional body. Theoretic models have shown that the propensity for metastases depends on the total number of cells of a tumor combined with the probability of each individual cell to disseminate [2]. This number of cells is probably better estimated by three-dimensional parameters such as tumor volume (TV) or surface area (TSA) (assuming that cells located at the advancing front of the tumor have a significant probability for dissemination). Depending on the shape of the tumor, there might be considerable variability in the TVs and TSAs within lesions of the same diameter. To exemplify this, a lesion 
measuring $30 \times 30 \times 30 \mathrm{~mm}$ has a TV of $14.14 \mathrm{~cm}^{3}$, a lesion measuring $30 \times 25 \times 20 \mathrm{~mm}$ one of $7.85 \mathrm{~cm}^{3}$ and a flat tumor measuring $30 \times 22 \times 15 \mathrm{~mm}$ a volume of only $5.18 \mathrm{~cm}^{3}$; the corresponding TSAs are as follows: 28.27 , 19.55 , and $15.48 \mathrm{~cm}^{2}$.

Our study addresses two important issues regarding three-dimensional pathologically measured parameters in breast cancer:

1. To analyze tumor shape and its variability, and to assess factors that influence tumor shape

2. To assess the prognostic impact of three-dimensional size measures: is TV and/or TSA, a more accurate predictor of axillary lymph node involvement than the established one-dimensional measurement (maximal diameter)?

Our study answers the question of whether routine pathological three-dimensional measurement of a tumor would have clinical relevance. At this time, the reporting of these measurements in the current pathology guidelines and protocols remains optional [3-5]. This study is reported according to the "REporting recommendations for tumor MARKer prognostic studies (REMARK)" [6].

\section{Patients and methods}

Patient characteristics and data collection (REMARK requirements 2, 4, 6 [6])

The data of 395 female breast cancer patients whose surgically removed primary carcinomas were pathologically examined at the Institute for Pathology of the University of Basel, Switzerland, between 1998 and 2005 form the basis of the current analysis. We restricted our study to the analysis of histologically proven unifocal invasive ductal carcinoma of limited size, in which the largest pathologically assessed tumor diameters measured between 11 and $40 \mathrm{~mm}$ (T1c, T2 $\leq 4 \mathrm{~cm})$. Three-dimensional measurements of each lesion, i.e. three PDs, were available for all tumors: the maximal diameter (MD) and two PD1, PD2; the data were organized as follows: $\mathrm{MD} \geq \mathrm{PD} 1 \geq \mathrm{PD} 2$. Pathologic lymph node status was known for all patients. We excluded patients who had multifocal/multicentric breast tumors or had tumors with clinical or pathological evidence of any kind of skin or chest wall involvement (T4 category). Patients who had preoperative neoadjuvant therapy were also excluded. Invasive lobular carcinomas and rare histologic subtypes with more favorable prognosis (e.g., tubular carcinoma, papillary carcinoma, medullary carcinoma) were not considered. In all patients included in the analysis, hormonal receptor assay, assessment of the HER-2/neu status and histolopathologic grading according to the Nottingham modification of the Scarff-BloomRichardson grading scheme had been performed. Furthermore, clinical data concerning age and tumor location (stratified as inner quadrant, outer quadrant and central region) were available. The study was carried out in accordance with the guidelines of the Ethics Committee of the University of Basel.

Data analysis (REMARK requirements 7-9 [6])

1. Tumor shape (eccentricity). To assess the "roundness" of a tumor, the "Eccentricity Factor" (EF), as previously described by Schwartz et al. [7], was employed as a measure of tumor shape. The EF describes the overall change in tumor shape based on the following formula (where MD is the maximal diameter and PD2 is the smaller of the two measured PDs:

$E F=\sqrt{1-(P D 2 / M D)^{2}}$

According to this formula, the EF values fall between zero and one: the higher the EF, the flatter the shape of the tumor. For example, a lesion measuring $30 \times 30 \times 30 \mathrm{~mm}$ would have an $\mathrm{EF}$ of 0 , whereas a lesion measuring $30 \times 25 \times$ $20 \mathrm{~mm}$ would have an EF of 0.75 (Fig. 1); the highest EF value in our study population was 0.98 with corresponding tumor measurements of $29 \times 14 \times 6 \mathrm{~mm}$.

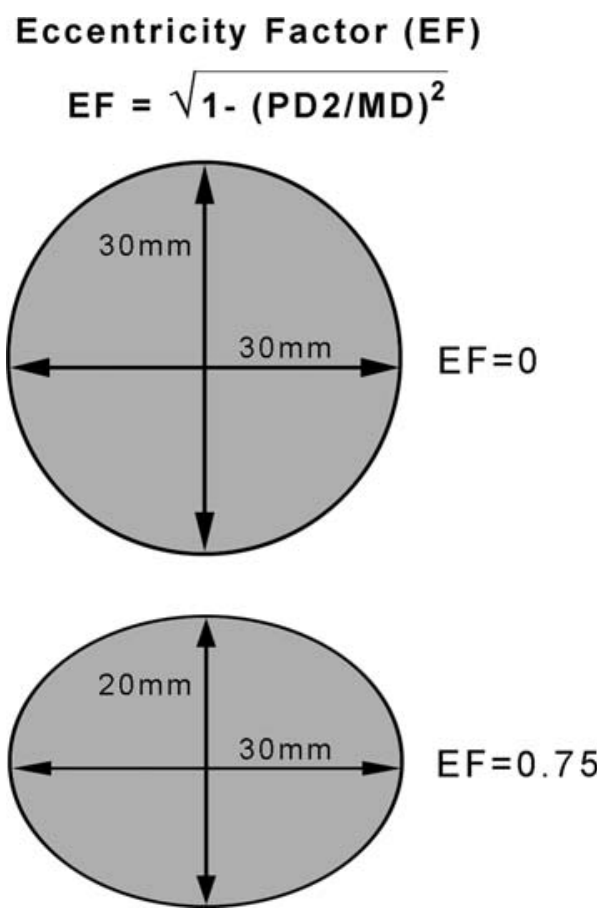

Fig. 1 Mathematical formula for the eccentricity factor and diagram illustrating two different eccentricity values. PD2: the smaller of both PDs; MD: maximal diameter 
2. Tumor volume (TV). For volume computation, the shape of the tumor was approximated to an ellipsoid. The volume was computed using the following equation (where MD is the maximal diameter and PD1/PD2 are diameters that are perpendicular to the $\mathrm{MD}$ and to each other in the three-dimensional space of the tumor body):

$V=1 / 6 \cdot \pi \cdot M D \cdot P D 1 \cdot P D 2$

3. Tumor surface area. The surface area was approximated by the following formula (where $p=$ 1.6075):

$S=4 \pi\left(\frac{\left(\frac{M D}{2}\right)^{P}\left(\frac{P D 1}{2}\right)^{P}+\left(\frac{M D}{2}\right)^{P}\left(\frac{P D 2}{2}\right)^{P}+\left(\frac{P D 1}{2}\right)^{P}\left(\frac{P D 2}{2}\right)^{P}}{3}\right)^{1 / p}$

4. Dependent variable. The dependent variable of our study was ALNI, the single most powerful prognostic factor for primary breast cancer. In our analyses, ALNI refers not to the nodal status (expressed as either the presence of negative or positive nodes), but rather the number of lymph nodes involved.

For every tumor analyzed in this study, we determined six specific morphologic values: largest diameter (MD), two PDs (PD1, PD2), TV, TSA and the EF.

Statistical analysis (REMARK requirements 10, 11 [6])

Ordinal linear regression was used to model the relation between tumor shape (EF) and selected morphologic features; to achieve approximately normal distribution for MD/ PD2, this variable was logarithmically transformed, and regression parameters were expressed as geometric mean ratios. To evaluate the influence of prognostic factors on ALNI, particularly MD, TV, TSA and EF, we used univariate and multivariate Poisson regression method [8]. This model was performed allowing for overdispersion. TV and TSA values were logarithmically transformed. Regression parameters are reported as risk ratios with $95 \%$ confidence intervals. In all tests, a $P$-value $<0.05$ was considered significant. To decide which of the three multivariate models including one of the highly confounded variables MD, TV and TSA has the best impact on ALNI, pseudo R-squared values according to Cox and Snell were calculated [9]. Statistical analyses were performed with R Development Core Team software, version 2.5.0 (Vienna, Austria).

\section{Results (REMARK requirements 12-17 [6])}

Clinicopathologic and morphologic characteristics of the 395 patients in the study are summarized in Table 1 .
Eccentricity, an expression of tumor shape in invasive ductal breast carcinoma, is depicted graphically by a box plot (Fig. 2). The most frequent tumor shape was scalene ellipsoid, i.e. an ellipsoid in which the lengths of all three semi-principal axes are different $(n=286,72.4 \%) .44$ patients $(11.1 \%)$ had tumors in which the largest diameter was repeated twice and the third dimension was a smaller number (oblate spheroid, e.g. $20 \times 20 \times 15 \mathrm{~mm}$ ). 56 patients $(14.2 \%)$ had dimensions in which there was one larger diameter and the smaller diameter was repeated twice (prolate spheroid, e.g. $20 \times 15 \times 15 \mathrm{~mm}$ ). Only nine carcinomas $(2.3 \%)$ had a spherical shape (all three diameters were identical; $\mathrm{EF}=0$ ). $50 \%$ of all lesions ranged between an $\mathrm{EF}$ of 0.61 and 0.83 . The median eccentricity value was 0.75 . This value means that the ratio between the MD and the smallest perpendicular diameter (PD2) is 1:0.67, or that PD2 is approximately 30\% less than the MD. Table 2 shows that established prognostic factors (histologic grade, estrogen receptor and HER-2/neu status) had no effect on tumor shape. Only the MD had a significant influence on the shape of the tumor: the larger the tumor, the flatter the shape $(P<0.001)$.

Using univariate analysis, maximal tumor diameter (MD), TV and TSA were highly significant predictors of ALNI; these variables had similar levels of prediction accuracy (MD: $P=0.0003$; TV: $P=0.0009$; TSA: $P<0.0001$; Table 3). Additionally, both perpendicular tumor diameters had significant association with ALNI (PD1: $P=0.0086$; PD2: $P=0.0106)$.

Multivariate analyses showed that MD, TV and TSA remained significant independent predictors of ALNI (MD: $P=0.0018 ; \quad$ TV: $\quad P=0.0109 ; \quad$ TSA: $\quad P=0.0009$; Table 4). Since these variables are highly confounded by the common value MD, they could not be calculated in a combined model [8]. Therefore, separate calculations were made for each variable. Table 4 shows the seven-variable model which includes MD in the calculation (in addition to MD/TV/TSA we chose to take six co-variables into consideration in the model: age, EF, tumor location, grading, estrogen receptor and HER-2/neu status). At the bottom of this table, the TV and TSA values are indicated. The values of the six co-variables in the calculations including TV and TSA are quite similar to the calculation including MD, therefore they were omitted from the table. The pseudo Rsquared values evaluating the three confounded variables showed the following ranking: MD: 0.42; TV: 0.39; TSA: 0.39 .

\section{Discussion (REMARK requirements 19, 20 [6])}

In the upcoming era of molecular and genetic characterization of breast cancer, the significance of traditional 
Table 1 Clinicopathologic and morphologic characteristics of the study group

\begin{tabular}{|c|c|c|c|c|}
\hline Variable & $\begin{array}{l}\text { Entire study } \\
\text { group }\end{array}$ & $\begin{array}{l}\text { Tumor size: } \\
11-20 \mathrm{~mm}\end{array}$ & $\begin{array}{l}\text { Tumor size: } \\
21-30 \mathrm{~mm}\end{array}$ & $\begin{array}{l}\text { Tumor size: } \\
\text { 31-40 mm }\end{array}$ \\
\hline Total number of cases $(\%)$ & $395(100)$ & $167(41.8)$ & $177(45.6)$ & $51(12.6)$ \\
\hline \multicolumn{5}{|l|}{ Age (yrs) } \\
\hline Mean & 61.7 & 59.8 & 63.1 & 63.2 \\
\hline Range & $31-88$ & $31-85$ & $31-88$ & $37-86$ \\
\hline \multicolumn{5}{|l|}{ Tumor diameter ${ }^{\mathrm{a}}$, mean $(\mathrm{mm})$} \\
\hline MD: maximal diameter & 22.7 & 16.3 & 25.0 & 36.0 \\
\hline PD1: perpendicular diameter 1 & 18.8 & 13.8 & 20.8 & 27.9 \\
\hline PD2: perpendicular diameter 2 & 14.9 & 11.4 & 16.5 & 20.7 \\
\hline \multicolumn{5}{|l|}{ Tumor volume $\left(\mathrm{cm}^{3}\right)$} \\
\hline Mean & 4.2 & 1.5 & 4.8 & 11.4 \\
\hline Range & $0.14-25.66$ & $0.14-4.19$ & $0.96-14.14$ & $3.14-25.66$ \\
\hline \multicolumn{5}{|l|}{ Tumor surface area $\left(\mathrm{cm}^{2}\right)$} \\
\hline Mean & 12.08 & 6.20 & 13.82 & 25.30 \\
\hline Range & $1.46-42.20$ & $1.46-12.57$ & $6.02-28.27$ & $12.98-42.20$ \\
\hline \multicolumn{5}{|l|}{ Eccentricity factor } \\
\hline Median & 0.75 & 0.70 & 0.75 & 0.82 \\
\hline Range & $0-0.98$ & $0-0.97$ & $0-0.98$ & $0.25-0.97$ \\
\hline \multicolumn{5}{|l|}{ Tumor location (\%) } \\
\hline Outer quadrants/central region & $295(74.7)$ & $120(71.9)$ & $135(76.3)$ & $40(78.4)$ \\
\hline Inner quadrants & $100(25.3)$ & $47(28.1)$ & $42(23.7)$ & $11(21.6)$ \\
\hline \multicolumn{5}{|l|}{ Grading $(\%)$} \\
\hline G1 & $52(13.2)$ & $35(21.0)$ & $17(9.6)$ & - \\
\hline $\mathrm{G} 2$ & $210(53.2)$ & $88(52.7)$ & $94(53.1)$ & $28(54.9)$ \\
\hline G3 & $133(33.6)$ & $44(26.3)$ & $66(37.3)$ & $23(45.1)$ \\
\hline Estrogen receptor positive $(\%)$ & $295(74.7)$ & $130(77.8)$ & $130(73.4)$ & $40(78.4)$ \\
\hline HER-2 neu positive (\%) & $75(19.0)$ & $24(14.4)$ & $38(21.5)$ & $13(25.5)$ \\
\hline \multicolumn{5}{|c|}{ Axillary lymph node involvement (\%) } \\
\hline Negative & $191(48.4)$ & $93(55.7)$ & $83(46.9)$ & $15(29.4)$ \\
\hline 1-3 lymph nodes & $148(37.4)$ & $59(35.3)$ & $68(38.4)$ & $21(41.2)$ \\
\hline 4-10 lymph nodes & $38(9.6)$ & $9(5.4)$ & $19(10.7)$ & $10(19.6)$ \\
\hline$>10$ lymph nodes & $18(4.6)$ & $6(3.6)$ & $7(4.0)$ & $5(9.8)$ \\
\hline
\end{tabular}

a The data were organized as follows: MD $\geq$ PD1 $\geq$ PD2

anatomic-morphologic prognostic determinants may very well take a back seat to these new indicators. Efforts should, however, be made to enhance the accuracy of these parameters. As before, the size of the tumor remains an outstanding prognostic factor. Characterization of a threedimensional body by a one-dimensional measurement only seems to be a rather inadequate method.

Studies concerning the three-dimensionality of breast lesions are mainly a domain of radiologists, where the tumors assessed are still in vivo [10, 11]. Only few studies have assessed the three-dimensional extent of surgically removed carcinomas themselves through pathological examination of the tumor specimen [12-14]; none of them, however, have addressed the prognostic significance of these measurements.
In our study, we analyzed the shape of pathologically examined breast carcinomas and described its variabilities. Furthermore, we assessed the prognostic impact of the three-dimensional size measurement of a lesion, namely TV and TSA and compared its predictive capacity with the established one-dimensional measurement, the MD. For improved uniformity and comparability, we analyzed only invasive ductal carcinoma, which comprises up to 80-90\% of all invasive breast carcinomas, thereby representing the single largest group of invasive breast carcinomas. These carcinomas typically form well-defined solid tumors and three-dimensional measurement of their size is relatively precise. Lobular carcinomas were excluded from analysis because in many cases, there is no grossly well-demarked mass, and therefore three dimensional assessment of size is 


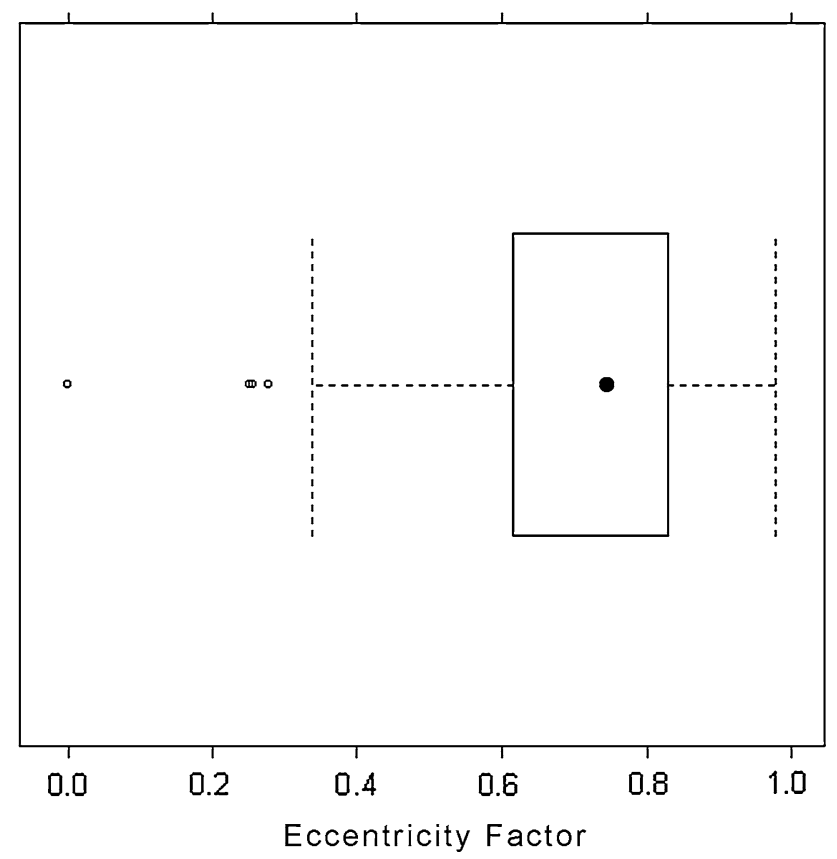

Fig. 2 Box plot for eccentricity of invasive ductal breast carcinoma. The box plot shows the median value (closed circle) and the $25 \%$ and $75 \%$ percentiles. The dashed lines label the $10 \%$ and $90 \%$ percentile, while the open circles represent outlying values

imprecise. More uncommon histologic subtypes were also excluded because they usually have a more favorable outcome (e.g., tubular, mucinous, medullary, papillary

Table 2 Associations between clinicopathologic/morphologic characteristics and tumor shape (eccentricity), univariate analysis

\begin{tabular}{llr}
\hline Variable & GMR $(95 \% \mathrm{CI})$ & $P$-value \\
\hline Maximal tumor diameter & $1.05(1.03-1.06)$ & $<0.001$ \\
Poor histologic grading (G3) & $0.98(0.91-1.06)$ & 0.640 \\
ER-negative status & $1.02(0.96-1.08)$ & 0.617 \\
c-erbB2 positive status & $1.01(0.95-1.08)$ & 0.703 \\
\hline
\end{tabular}

GMR: Geometric Mean Ratio; CI: Confidence Interval carcinomas) compared to invasive ductal and lobular carcinomas and the number of cases was so small that they cannot even be reasonably analyzed as independent subgroups.

Our data show that most breast carcinomas share a similar scalene ellipsoid shape and that the variability of eccentricity is relatively low. Despite certain variations in tumor shape and at times considerable variabilities in TV and TSA with similar MD (or within subgroups of diameters with a range of $10 \mathrm{~mm}$, see Table 1), there is no evidence that the three-dimensional pathologic measurements (TV/TSA) are more precise prognostic predictors of ALNI compared to MD alone. Of note, even the usually smaller PDs still have prognostic significance. This implies that imprecision of pathologic measurement of the largest dimension of the tumor might not be so critical.

Even in subgroups with tumors at extremes of shape (namely spherical or excessively flattened), three-dimensional measurement is not a more powerful predictor than MD. We expected this to be the case with flattened tumors, which have correspondingly lower TV and TSA. A possible explanation could have been that these tumors had more often characteristics that are associated with tumor aggressiveness (e.g., poor grading, hormonal receptor negative and HER-2 neu positive status). However, this was not the case; only MD had a significant influence on the shape of the tumor. The fact that tumors become flatter with increasing size probably has to do with their blood supply. Wapnir et al. demonstrated an inverse relationship between microvessel counts and TV [13].

The finding that three-dimensional measurements are not more powerful predictors of prognosis than tumor size alone might rather support the notion that only a small subpopulation of tumor cells in a whole tumor population has metastatic potential, and that this might not be dependent on total TV or total amount of tumor cells, but possibly on the individual quantity of metastases initiating
Table 3 Associations between clinicopathologic/morphologic characteristics and axillary lymph node involvement: univariate analysis

OR: Odds Ratio; CI: Confidence Interval

a The data were organized as follows: $\mathrm{MD} \geq \mathrm{PD} 1 \geq \mathrm{PD} 2$

\begin{tabular}{llr}
\hline Variable & OR $(95 \%$ CI $)$ & $P$-value \\
\hline Age & $1.02(0.88-1.19)$ & 0.788 \\
Tumor diameter $^{\mathrm{a}}$ & & \\
$\quad$ MD: maximal diameter & $1.28(1.12-1.45)$ & 0.0003 \\
$\quad$ PD1: perpendicular diameter 1 & $1.22(1.05-1.41)$ & 0.0086 \\
$\quad$ PD2: perpendicular diameter 2 & $1.26(1.05-1.49)$ & 0.0106 \\
Tumor volume & $1.46(1.17-1.82)$ & 0.0009 \\
Tumor surface area & $1.79(1.28-2.50)$ & $<0.0001$ \\
Eccentricity factor & $0.94(0.33-3.02)$ & 0.920 \\
Tumor localization: outer quadrants/ central region & $1.43(0.87-2.47)$ & 0.177 \\
Poor histologic grading (G3) & $2.73(1.26-7.47)$ & 0.0249 \\
ER-negative status & $1.59(1.05-2.37)$ & 0.0240 \\
HER-2 neu positive status & $2.04(1.34-3.04)$ & $<0.0001$ \\
\hline
\end{tabular}


Table 4 Multivariate Poisson regression analyses for factors that influence axillary lymph node involvement: the model was calculated separately for each of the variables maximal tumor diameter, tumor volume and surface area

OR: Odds ratio; CI: Confidence interval

\begin{tabular}{lll}
\hline Variable & OR $(95 \% \mathrm{CI})$ & $P$-value \\
\hline Maximal tumor diameter & $1.23(1.08-1.40)$ & 0.0018 \\
Age & $1.02(0.88-1.17)$ & 0.831 \\
Eccentricity factor & $0.59(0.22-1.72)$ & 0.311 \\
Tumor localization: outer & $1.39(0.89-2.28)$ & 0.165 \\
$\quad$ quadrants/central region & & \\
Poor histologic grading (G3) & $1.80(0.84-4.68)$ & 0.174 \\
ER-negative status & $1.28(0.85-1.90)$ & 0.234 \\
c-erbB2 positive status & $1.66(1.09-2.48)$ & 0.0164 \\
Tumor volume & $1.33(1.07-1.66)$ & 0.0109 \\
Tumor surface area & $1.55(1.12-2.16)$ & 0.0009 \\
\hline
\end{tabular}

tumor stem cells or the gain of metastatic possibility of these cells over time [15]. Furthermore, there is a mathematical explanation for the statistical lack of higher impact of three-dimensionality: the multiplication of three metric variables results in more variability of the predictor, and this leads to higher variability of the data and to blurred statistical trends.

According to the current pathology guidelines and protocols, a three-dimensional measurement of breast carcinomas remains optional [3-5], with the comment in the College of American Pathologists protocol that this has not yet been validated [4]. Our data do not support the assumption that three-dimensional measurement has an improved prognostic capability in comparison with traditional one-dimensional measurement. Nevertheless, threedimensional pathologic measurement of a tumor provides useful information. It allows a more exact morphologic description of the lesion compared to the assessment of MD alone. Furthermore, a routinely performed threedimensional measurement helps the pathologist in identifying the plane of the longest tumor axis and to assess the truly largest dimension.

\section{References}

1. Chang J, Hilsenbeck S (2004) Prognostic and predictive markers. In: Harris J, Lippman M, Morrow M, Osborne K (eds) Diseases of the breast, 3rd edn. Lippincott Williams \& Wilkins, Philadelphia

2. Michaelson JS, Silverstein M, Wyatt J et al (2002) Predicting the survival of patients with breast carcinoma using tumor size. Cancer 95(4):713-723

3. Pathology Reporting of Breast Disease (NHSBSP Publication No 58) (2005) NHS cancer screening programmes jointly with the Royal College of Pathologists. London
4. Fitzgibbons P, Connolly J, Page D, for College of American Pathologists (2005) Breast 2005 surgical pathology cancer case summary, 1/05 update. Available from URL: www.cap.org/apps/ docs/cancer_protocols/2005/breast05_pw.pdf

5. Wells C (2006) Quality assurance guidelines for pathology. In: Perry N, Broeders M, de Wolf C, Törnberg S, Holland R, von Karsa L (eds) European guidelines for quality assurance in breast cancer screening and diagnosis, 4th edn. Office for Official Publications of the European Communities, Luxembourg

6. McShane LM, Altman DG, Sauerbrei W, Taube SE, Gion M, Clark GM (for the Statistics Subcommittee of the NCI-EORTC Working Group on Cancer Diagnostics) (2006) REporting recommendations for tumor MARKer prognostic studies (REMARK). Breast Cancer Res Treat 100(2):229-235

7. Schwartz LH, Colville JA, Ginsberg MS et al (2006) Measuring tumor response and shape change on CT: esophageal cancer as a paradigm. Ann Oncol 17(6):1018-1023

8. Agresti A (2007) An introduction to categorical data analysis, 2nd edn. Wiley, Hoboken

9. Freese J, Long J (2006) Regression models for categorical dependent variables using stata, 2nd edn. Stata Pres, College Station

10. Cho N, Moon WK, Cha JH et al (2006) Differentiating benign from malignant solid breast masses: comparison of two-dimensional and three-dimensional US. Radiology 240(1):26-32

11. Schnall MD, Blume J, Bluemke DA et al (2006) Diagnostic architectural and dynamic features at breast MR imaging: multicenter study. Radiology 238(1):42-53

12. Andea AA, Bouwman D, Wallis T, Visscher DW (2004) Correlation of tumor volume and surface area with lymph node status in patients with multifocal/multicentric breast carcinoma. Cancer 100(1):20-27

13. Wapnir IL, Barnard N, Wartenberg D, Greco RS (2001) The inverse relationship between microvessel counts and tumor volume in breast cancer. Breast J 7(3):184-188

14. Wapnir IL, Wartenberg DE, Greco RS (1996) Three dimensional staging of breast cancer. Breast Cancer Res Treat 41(1):15-19

15. Sleeman JP, Cremers N (2007) New concepts in breast cancer metastasis: tumor initiating cells and the microenvironment. Clin Exp Metastasis 24(8):707-715 